В.А. Головачева ${ }^{\boxplus}$, ORCID: 0000-0002-2752-4109, e-mail: xoxo.veronicka@gmail.com

B.A. Парфенов, ORCID: 0000-0002-1992-7960

Первый Московский государственный медицинский университет имени И.М. Сеченова (Сеченовский Университет); 119991, Россия, Москва, ул. Трубецкая, д. 8, стр. 2

\title{
Резюме
}

Острая неспецифическая пояснично-крестцовая боль (ПКБ) - одна из самых частых причин обращения к врачу. Данное заболевание имеет благоприятный прогноз, однако при неадекватном лечении боль в спине может приобрести хроническое течение. В связи с этим своевременная диагностика и адекватное лечение острой неспецифической ПКБ имеют большое клиническое значение. Диагноз острой неспецифической ПКБ ставится после исключения дискогенной радикулопатии, поясничного стеноза и других специфических причин боли в спине. В 2018 г. был проведен обзор 15 клинических руководств по лечению острой неспецифической ПКБ, сделано заключение, что они совпадают по основным терапевтическим принципам. Информирование пациента о благоприятном прогнозе боли, рекомендация поддерживать активный образ жизни и оптимальная фармакотерапия - основа эффективного лечения острой неспецифической ПКБ. Для облегчения боли в спине эффективны нестероидные противовоспалительные средства (НПВС). Выбор препарата НПВС осуществляется в зависимости от сопутствующих заболеваний пациента и риска развития побочных эффектов. При недостаточной эффективности НПВС возможно добавление препарата из группы миорелаксантов. Новое направление в фармакотерапии острой неспецифической ПКБ комбинация НПВС и витаминов группы В (тиамин, пиридоксин, цианокобаламин). Обсуждаются результаты клинических исследований эффективности и безопасности комбинации витаминов группы В и НПВС, преимущества назначения данной комбинации при ПКБ. Анализируется применение препарата Мильгамма, содержащего витамины группы В, в качестве коанальгетика в комбинации с НПВС при острой неспецифической боли в спине.

Ключевые слова: острая неспецифическая пояснично-крестцовая боль, острая боль в нижней части спины, терапия, нестероидные противовоспалительные средства, витамины группы В, Мильгамма

Для цитирования: Головачева В.А., Парфенов В.А. Лечение острой неспецифической пояснично-крестцовой боли: современные рекомендации и новые подходы в фармакотерапии. Медицинский совет. 2019;(18):34-41. doi: 10.21518/2079-701X-2019-18-34-41.

Конфликт интересов: авторы заявляют об отсутствии конфликта интересов.

\section{Management of acute nonspecific lumbosacral pain: modern guidelines and new approaches in pharmacotherapy}

Veronika A. Golovacheva, ORCID: 0000-0002-2752-4109, e-mail: xoxo.veronicka@gmail.com

Vladimir A. Parfenov, ORCID: 0000-0002-1992-7960

I.M. Sechenov First Moscow State Medical University (Sechenov University); 8, Bldg. 2, Trubetskaya St., Moscow, 119991, Russia

\begin{abstract}
Acute nonspecific lumbosacral pain (LSP) is one of the most common reasons people visit their doctor. This disease has a favourable prognosis, however, the low back pain can progress to a chronic course, if the treatment is inadequate. In this regard, timely diagnosis and adequate treatment of acute nonspecific LSP are of great clinical importance. The diagnosis of acute non-specific LSP is established after discogenic radiculopathy, lumbar stenosis and other specific causes of back pain have been excluded. 15 clinical guidelines for the treatment of acute nonspecific LSP were reviewed in 2018, and it was concluded that they match in basic therapeutic principles. The basis for effective treatment of acute nonspecific LSP includes informing the patient about a favourable prognosis of pain, the recommendation to maintain an active lifestyle and optimal pharmacotherapy. Nonsteroidal anti-inflammatory drugs (NSAIDs) are effective in relieving the low back pain. The choice of NSAIDs depends on the concomitant diseases of the patient and the risk of side effects. If NSAIDs provide poor efficacy, a drug from the muscle relaxants group can be added. NSAIDs combined with B vitamins (thiamine, pyridoxine, cyanocobalamin) is a new direction in the pharmacotherapy of acute non-specific LSP. The article discusses the results of clinical trials of the efficacy and safety of NSAIDs combined with B vitamins, the advantages of this combination in patients with LSP.The authors analyse the use of B vitamins-containing Milgamma as a co-analgesic drug combined with NSAIDs in acute nonspecific back pain.
\end{abstract}

Keywords: acute nonspecific lumbosacral pain, acute pain in the lower back, therapy, non-steroidal anti-inflammatory drugs, B vitamins, Milgamma

For citation: Golovacheva V.A., Parfenov V.A. Treatment of acute nonspecific lumbosacral pain: modern guidelines and new approaches in pharmacotherapy. Meditsinskiy sovet = Medical Council. 2019;(18):34-41. (In Russ.) doi: 10.21518/2079-701X-2019-18-34-41.

Conflict of interest: The authors declare no conflict of interest. 


\section{ВВЕДЕНИЕ}

Острая пояснично-крестцовая боль (ПКБ) - это боль, которая локализуется между двенадцатой парой ребер и ягодичными складками и продолжается не более 4 недель [1]. ПКБ - одна из самых частых жалоб пациентов на приеме у невролога и одна из самых распространенных причин временной нетрудоспособности [2]. Из-за ПКБ 6-9\% взрослого населения в течение года обращаются за медицинской помощью, а в течение жизни ПКБ возникает у $70 \%$ людей [3].

В 90-95\% случаев острая ПКБ вызвана скелетномышечной патологией, которая расценивается как неспецифическая [4]. При скелетно-мышечной (неспецифической) ПКБ нет признаков радикулопатии, поясничного стеноза и отсутствуют какие-либо специфические причины боли: переломы позвонков, новообразования и другие заболевания, приводящие к развитию боли в спине [5]. Наиболее часто к анатомическим источникам неспецифической (скелетно-мышечной) ПКБ относятся такие структуры, как межпозвоночный диск, фасеточные суставы, крестцово-подвздошные суставы, мышцы спины и связки $[1,4]$. Однако при неспецифической ПКБ часто не удается установить точные источники боли. В соответствии с современными представлениями о патогенезе и терапии неспецифической (скелетно-мышечной) ПКБ, точное определение анатомических источников боли при данном заболевании не улучшает течение и прогноз [6]. В российской практике распространен ошибочный взгляд на остеохондроз как причину боли в спине [7]. Ведущие мировые эксперты подчеркивают, что остеохондроз - это естественный процесс дегенерации (старения) структур позвоночника, который наблюдается в разной степени у всех людей, прогрессирует с возрастом, но не является причиной неспецифической боли в спине [8].

В 3-10\% случаев неспецифическая ПКБ сохраняется более 12 недель, поэтому расценивается как хроническая [4, 6]. У пациентов с острой неспецифической ПКБ хронизация боли происходит при неадекватном лечении, длительном постельном режиме, ограничительном поведении (избегании физической активности, чрезмерном ограничении движений в области пояснично-крестцового отдела позвоночника, ношении поясничных корсетов и бандажей), психологических расстройствах (сниженном фоне настроения, тревоге, катастрофизации боли). В особых, более редких ситуациях хронизация ПКБ может возникать, если пациент заинтересован в длительной нетрудоспособности, аггравирует симптомы или есть рентное отношение к болезни [9-12].

Своевременная диагностика острой неспецифической ПКБ, информирование пациента о благоприятном прогнозе заболевания и современные рекомендации по лечению позволят эффективно облегчить боль, повысить трудоспособность, ускорить выздоровление пациента и его возвращение к повседневной активности, предупредить хронизацию ПКБ [9-12].
ОСНОВНЫЕ ПРИНЦИПЫ ДИАГНОСТИКИ ОСТРОЙ НЕСПЕЦИФИЧЕСКОЙ ПОЯСНИЧНО-КРЕСТЦОВОЙ БОли

Острая неспецифическая ПКБ диагностируется на основании данных анамнеза и клинического обследования [1]. В МКБ-10 неспецифическая ПКБ соответствует рубрикам М.54.5 (боль внизу спины, люмбалгия) и M54.4 (люмбаго с ишиасом) [8]. Диагноз острой неспецифической ПКБ устанавливается после исключения дискогенной радикулопатии, стеноза спинномозгового канала и других специфических причин боли в спине [4]. В беседе с пациентом и при проведении осмотра необходимо исключить наличие «красных флажков» - знаков и симптомов, настораживающих врача в отношении специфической боли в спине. При обнаружении «красных флажков» необходимо проведение соответствующих лабораторных и инструментальных исследований для установления причины боли в спине (табл.) [8].

Если пациент молодого или среднего возраста (18-50 лет), у него нет «красных флажков», клиническая картина ПКБ типична для неспецифической (скелетно-мышечной) боли, то проведение дополнительных методов обследования не требуется. Если консервативное лечение острой неспецифической ПКБ не улучшает состояние пациента в течение 4 недель, боль усилилась или появились новые симптомы, то необходимо проведение рентгеновской КТ или МРТ поясничнокрестцового отдела позвоночника $[8,13]$.

\section{МЕЖДУНАРОДНЫЕ И НАЦИОНАЛЬНЫЕ РУКОВОДСТВА ПО ЛЕЧЕНИЮ ОСТРОЙ НЕСПЕЦИФИЧЕСКОЙ ПОЯСНИЧНО-КРЕСТЦОВОЙ БОЛИ}

Последние рекомендации по лечению острой неспецифической ПКБ были написаны экспертами из Великобритании, Дании, США, а также российскими специалистами из Российского общества по изучению боли $[1,8,13,14]$. В 2018 г. был проведен обзор клинических практических руководств по ведению пациентов с пояснично-крестцовой болью из 15 стран мира [15]. Было выяснено, что все современные руководства совпадают по основным принципам лечения острой неспецифической ПКБ. Во всех руководствах отмечается благоприятный прогноз острой неспецифической ПКБ: в большинстве случаев боль регрессирует в течение нескольких дней или недель. Рекомендуется рассказать пациенту о хорошем прогнозе острой неспецифической ПКБ, доброкачественном характере заболевания, высокой вероятности быстрого выздоровления, целесообразности поддерживать активный образ жизни и при возможности продолжать профессиональную, социальную и бытовую деятельность. Для уменьшения боли рекомендуются нестероидные противовоспалительные средства (НПВС). 
Таблица. «Красные флажки» при пояснично-крестцовой боли, их возможные причины и врачебная тактика

Table. «Red flags» for lumbosacral pain: possible causes and medical tactics

\begin{tabular}{|c|c|c|}
\hline «Красные флажки» & Возможные причины боли в спине & Врачебная тактика \\
\hline $\begin{array}{l}\text { Злокачественное новообразование в анамнезе, } \\
\text { неясная потеря веса, возраст пациента > } 50 \text { лет, } \\
\text { появление или усиление боли в покое, в ночное } \\
\text { время }\end{array}$ & Злокачественное новообразование & $\begin{array}{l}\text { КТ или МРТ позвоночника, в некоторых случаях - } \\
\text { сцинтиграфия позвоночника, консультация онко- } \\
\text { лога }\end{array}$ \\
\hline $\begin{array}{l}\text { Лихорадка, недавно перенесенное инфекцион- } \\
\text { ное заболевание, парентеральное употребление } \\
\text { наркотиков }\end{array}$ & Инфекционное поражение позвонков или дисков & КТ или МРТ позвоночника \\
\hline $\begin{array}{l}\text { Тазовые расстройства, парезы конечностей, ане- } \\
\text { стезия промежности }\end{array}$ & Синдром поражения корешков конского хвоста & $\begin{array}{l}\text { КТ или МРТ позвоночника, консультация невро- } \\
\text { лога, нейрохирурга }\end{array}$ \\
\hline $\begin{array}{l}\text { Наличие признаков остеопороза, прием кортико- } \\
\text { стероидов, недавняя травма спины }\end{array}$ & Компрессионный перелом позвоночника & $\begin{array}{l}\text { Рентгенография, КТ или МРТ позвоночника, ден- } \\
\text { ситометрия }\end{array}$ \\
\hline $\begin{array}{l}\text { Утренняя скованность, пробуждение во второй } \\
\text { половине ночи из-за боли }\end{array}$ & Спондилоартрит & Консультация ревматолога \\
\hline $\begin{array}{l}\text { Наличие пульсирующего образования в брюшной } \\
\text { полости }\end{array}$ & Аневризма брюшного отдела аорты & $\begin{array}{l}\text { Ультразвуковое исследование, консультация } \\
\text { хирурга }\end{array}$ \\
\hline $\begin{array}{l}\text { Сегментарно-корешковый тип нарушения чув- } \\
\text { ствительности, выраженная или нарастающая } \\
\text { слабость в ногах. } \\
\text { Положительные симптомы натяжения спинномоз- } \\
\text { говых корешков }\end{array}$ & Грыжа диска и (или) поясничный стеноз & $\begin{array}{l}\text { КТ или МРТ позвоночника, консультация невро- } \\
\text { лога, нейрохирурга }\end{array}$ \\
\hline
\end{tabular}

\section{ПРИНЦИПЫ ЛЕЧЕНИЯ ОСТРОЙ НЕСПЕЦИФИЧЕСКОЙ ПОЯСНИЧНО-КРЕСТЦОВОЙ БОЛИ}

В соответствии с международными и национальными руководствами лечение острой неспецифической ПКБ основывается на трех принципах: 1) информировании пациента о благоприятном прогнозе заболевания; 2) рекомендации сохранять активный образ жизни; 3) фармакотерапии, направленной на облегчение боли $[1,8,13-15]$. К эффективной фармакотерапии острой неспецифической ПКБ относятся препараты из группы НПВС: неселективные ингибиторы циклооксигеназы-2 (ЦОГ-2), селективные ингибиторы ЦОГ-2 (коксибы) [15]. Выбор НПВС индивидуален и зависит от сопутствующих заболеваний пациента и риска развития побочных эффектов со стороны желудочно-кишечного тракта, сердечно-сосудистой системы, печени и почек [1, 8, 13-15]. К фармакотерапии острой неспецифической ПКБ также относятся препараты из группы миорелаксантов [15]. При болях в спине наиболее часто используются такие миорелаксанты, как тизанидин и баклофен [16]. Нет убедительных данных о преимуществе одного миорелаксанта перед другим в лечении острой неспецифической ПКБ $[8,15]$. Результаты клинических исследований о терапевтическом преимуществе комбинации НПВС и миорелаксанта перед монотерапией НПВС противоречивы [17-19]. Назначение миорелаксанта рекомендуется при недостаточной эффективности монотерапии НПВС [20].

Как показывают клинические наблюдения, для лечения острой неспецифической ПКБ часто применяются и другие методы лечения: вытяжение, иглорефлексотерапия, различные методы физиотерапии, ношение специ- альных поясов и корсетов, использование ортопедических стелек и обуви, мануальная терапия, массаж [8-12]. Однако перечисленные методы не обладают достоверной эффективностью, а в ряде случаев могут быть небезопасны для пациента [8, 15]. Вытяжение при острой неспецифической ПКБ может ухудшать ее течение. Иглорефлексотерапия, различные методы физиотерапии, ношение специальных поясов и корсетов на области поясницы, использование ортопедических стелек и обуви не признаются эффективными методами лечения острой неспецифической ПКБ $[8,15]$. Мануальная терапия может назначаться пациентам с острой неспецифической ПКБ, особенно при недостаточной эффективности стандартной терапии, однако данный вопрос остается дискутабельным [8, 15, 21, 22].

В практической деятельности часто возникают трудности лечения пациентов с острой неспецифической ПКБ, связанные с несколькими проблемами: недостаточным эффектом НПВС в средних терапевтических дозах; развитием побочных эффектов при назначении НПВС, особенно в высоких терапевтических дозах или при приеме более 7 дней; наличием противопоказаний для добавления миорелаксанта к НПВС или развитием побочных эффектов от миорелаксанта [23]. В соответствии с перечисленными клиническими ситуациями возникает необходимость поиска новых вариантов лекарственной терапии острой неспецифической ПКБ. Практический интерес представляют препараты, которые можно комбинировать с НПВС. К задачам комбинированной терапии относится усиление обезболивающего эффекта НПВС, сокращение доз и продолжительности приема НПВС, снижение риска развития побочных эффектов от терапии 
НПВС, поиск альтернативных вариантов лечения при невозможности добавлять миорелаксанты к НПВС [23]. На сегодняшний день при острой неспецифической ПКБ в качестве коанальгетиков для НПВС рассматриваются витамины группы В [24]. Препараты, содержащие витамины группы В, давно применяются в неврологической практике; относительно недавно стали обсуждать новые свойства и возможности применения витаминов группы В при болевых синдромах.

\section{ПРИМЕНЕНИЕ ВИТАМИНОВ ГРУППЫ В В НЕВРОЛОГИЧЕСКОЙ ПРАКТИКЕ}

В неврологической практике широко применяются витамины группы В, при этом чаще всего используются комбинированные препараты, содержащие тиамин (витамин В1), пиридоксин (В6), цианокобаламин (В12) [25-30]. Перечисленные витамины участвуют в таких нейропластических процессах, как формирование новых отростков нейронов, ремиелинизация, синаптогенез [31, 32]. Тиамин оказывает существенное влияние на процессы регенерации поврежденных нервных волокон, участвует в энергетических процессах в нервных клетках и поддерживает нормальную функцию аксоплазматического тока. Пиридоксин поддерживает синтез транспортных белков в аксонах, участвует в биосинтезе многих медиаторов и оказывает антиоксидантное действие [33]. Также установлено, что комбинация различных витамеров пиридоксина улучшает эндоневральный кровоток, угнетая агрегацию тромбоцитов [34]. Кобаламин (производное цианокобаламина) участвует в биохимических процессах, обеспечивающих нормальный синтез миелина, что имеет большое значение при демиелинизации нервных волокон.

Одновременное применение нескольких препаратов, содержащих отдельные витамины группы В, может технически усложнять процесс лечения. В связи с этим наличие препарата, содержащего комплекс витаминов В1, В6 и B12, практически ценно. Применение комбинированных препаратов витаминов группы В облегчает процесс проведения терапии и способствует повышению приверженности пациента к лечению [23].

Мильгамма - один из наиболее изученных препаратов, содержащих комбинацию витаминов группы В [24]. Мильгамма - препарат в форме раствора, в одной ампуле (2 мл) содержится 100 мг тиамина, 100 мг пиридоксина, 1 мг цианокобаламина, 20 мг лидокаина.

Обычно лидокаин входит в состав лекарственных растворов для внутримышечных инъекций, если введение самого лекарственного вещества болезненно. Например, для безболезненного введения пенициллина в состав раствора входит лидокаин [35]. Внутримышечное введение витаминов группы В - болезненный процесс для пациентов. Наличие лидокаина в составе комбинированного препарата витаминов группы В (Мильгамма) позволяет проводить инъекции безболезненно. Данное свойство препарата имеет большую практическую значимость и повышает приверженность пациентов к лечению.

\section{ИЗУЧЕНИЕ АНАЛЬГЕТИЧЕСКИХ СВОЙСТВ ВИТАМИНОВ ГРУППЫ В}

В неврологической практике распространено мнение, что цель назначения витаминов группы В при боли - это активация метаболических процессов в нервных волокнах, синтез миелина [36]. Действие витаминов группы В в высоких дозах отличается от их действия в физиологических дозах, которые ежедневно поступают с пищей [37]. Авторы экспериментальных исследований подчеркивают тот факт, что витамины группы В в высоких дозах уменьшают интенсивность боли за счет влияния на ее патогенетические механизмы [38, 39].

В 1980-х гг. были проведены экспериментальные исследования на лабораторных животных, показавшие противоболевую и противовоспалительную активность витаминов группы В [40]. В эксперименте с «воспалительной» моделью ноцицептивной боли определяли антиноцицептивный эффект при пероральном введении диклофенака, его комбинации с витаминами В1, B6, B12 и при введении только витаминов группы В. Авторы показали антиноцицептивный эффект комбинации В1, B6, B12 и предположили, что комбинация витаминов группы В ингибирует синтез воспалительных медиаторов (алгогенов) [36]. В другом эксперименте изучали действие тиамина на ноцицептивную и невропатическую боль. Тиамин значительно уменьшал ноцицептивную и невропатическую боль, сопровождавшуюся тактильной аллодинией [37]. В эксперименте на мышах продемонстрировано, что тиамин уменьшает интенсивность ноцицептивной воспалительной боли как острого, так и хронического течения. При этом отмечен дозозависимый обезболивающий эффект тиамина: чем выше доза тиамина, тем сильнее обезболивающее действие [38]. По результатам сравнительных экспериментальных исследований авторы сделали вывод, что комбинированное введение витаминов В1, В6 и В12 превосходит введение данных витаминов по отдельности в отношении противоболевого и противовоспалительного эффекта [39]. Показано, что комплексное применение витаминов B1, В6 и В12 усиливает действие противоболевых нейромедиаторов: норадреналина и серотонина. Также в экспериментальном исследовании было обнаружено подавление ноцицептивных ответов не только в заднем роге, но и в зрительном бугре [38, 41].

В дальнейшем противоболевой эффект витаминов группы В и повышение эффективности терапии за счет комбинированного применения НПВС и витаминов группы В были продемонстрированы в клинических исследованиях, включавших пациентов с болью в спине [42-44]. Витамины группы В рассматриваются как коанальгетики для НПВС при острой неспецифической ПКБ [27]. Комбинированная фармакотерапия НПВС и витаминов группы В обсуждается как новый дополнительный вариант лечения пациентов с острой неспецифической ПКБ. Данный подход особенно интересен в тех случаях, когда эффект от монотерапии НПВС недостаточен, имеются противопоказания к назначению НПВС и миорелаксантов или возникают побочные эффекты от стандартной фармакотерапии. 


\section{КЛИНИЧЕСКИЙ ОПЫТ ПРИМЕНЕНИЯ ВИТАМИНОВ ГРУППЫ В У ПАЦИЕНТОВ С ОСТРОЙ НЕСПЕЦИФИЧЕСКОЙ ПОЯСНИЧНОЙ БОЛЬЮ}

Первые клинические исследования эффективности витаминов группы В при боли в спине были проведены в конце 1980-х и начале 1990-х гг. [43-45]. По дизайну данные исследования были рандомизированными, двойными слепыми, включали небольшое количество пациентов с болью в нижней части спины. В данных исследованиях всех пациентов разделяли на две группы: 1) группа, получающая монотерапию диклофенаком; 2) группа, получающая комбинированную терапию диклофенаком и фиксированной комбинацией витаминов В1, B6, В12. Получены данные, что комбинированное лечение по эффективности превосходит монотерапию диклофенаком. Преимущества комбинированной терапии - возможность сократить продолжительность приема НПВС и тем самым снизить частоту развития побочных эффектов.

В связи с положительными результатами небольших клинических исследований и их практической значимостью в дальнейшем были проведены более крупные рандомизированные исследования. Так, в бразильское рандомизированное двойное слепое исследование DOLOR было включено 372 амбулаторных пациента с острой ПКБ в возрасте от 18 до 65 лет [46]. Продолжительность болевого синдрома не превышала трех дней. Методом рандомизации 185 пациентов получали монотерапию диклофенаком, 187 пациентов - комбинированную терапию диклофенаком и витаминами группы В. Пациенты из группы монотерапии получали по 50 мг диклофенака в таблетках 2 раза в день (во время завтрака и ужина). Пациенты из группы комбинированной терапии получали таблетки, содержащие 50 мг диклофенака, 50 мг тиамина, 50 мг пиридоксина, 1 мг цианокобаламина, дважды в день (во время завтрака и ужина). Через три дня лечения значимое облегчение боли и возможность завершить прием препаратов отметили 46,5\% пациентов из группы комбинированного лечения и только 29,7\% пациентов - из группы монотерапии. Данное количественное различие между группами $(16,8 \%)$ было достоверно значимо ( $p=0,0005)$ и демонстрировало преимущество комбинированной терапии в скорости облегчения боли. Через 5 дней лечения из 123 пациентов, продолжавших прием препаратов, значимое облегчение боли и возможность завершить прием препаратов отметили 82\% пациентов из группы комбинированного лечения и только 43\% пациентов из группы монотерапии, что снова продемонстрировало достоверно значимое клиническое превосходство комбинированной терапии над монотерапией ( $<$ 0,000). Через семь дней лечения 14 из 16 (87,5\%) пациентов, продолжающих получать комбинированную терапию, отметили значимое облегчение боли и завершили лечение. Только у двух (12,5\%) пациентов из группы комбинированного лечения терапевтический эффект не был достигнут. Так, через семь дней лечения 59 из 68 (86,76\%) пациентов, продолжающих монотерапию диклофенаком, достигли значимого облегчения боли и завершили терапию. Девять (13,23\%) пациентов, получающих монотерапию диклофенаком, не достигли терапевтического эффекта. Авторы заключили, что достижение значимого облегчения боли в группе монотерапии диклофенаком заняло больше времени. Комбинированная терапия позволяет значимо сократить продолжительность приема фармакотерапии. Почти половина (46,5\%) пациентов, получающих комбинированную фармакотерапию, имели значимое облегчение уже через 3 дня лечения.

В России также были проведены клинические исследования эффективности комбинированной терапии НПВС и витаминов группы В при острой боли в спине [23]. В рандомизированном открытом сравнительном исследовании, включавшем 60 пациентов с острой ПКБ, проводилась оценка эффективности препарата Мильгамма в виде раствора для внутримышечных инъекций [47]. Методом рандомизации все пациенты были разделены на три группы: 1) монотерапия диклофенаком (75 мг, в форме внутримышечных инъекций, 1 раз в день); 2) монотерапия Мильгаммой (2 мл препарата, в форме внутримышечных инъекций, 1 раз в день); 3) комбинированная терапия диклофенаком (75 мг, внутримышечно) и Мильгаммой (2 мл, внутримышечно) 1 раз в день. Лечение проводилось амбулаторно, до 10 дней. Авторы отметили, что достоверно значимое снижение среднего значения интенсивности боли по визуально-аналоговой шкале (ВАШ) отмечалось со второго дня лечения в группах монотерапии и уже с первого дня лечения - в группе комбинированного лечения. Через 10 дней лечения регресс боли в спине отметили 45\% пациентов в группе Мильгаммы, 35\% - в группе диклофенака, 70\% - в группе комбинированного лечения. Частота побочных эффектов (боли в области эпигастрия, диарея) была выше в группах пациентов, принимавших диклофенак в качестве монотерапии и в комбинации с Мильгаммой. Среди пациентов, получающих монотерапию диклофенаком, побочные эффекты были отмечены в 35\% случаев. В группе комбинированной терапии побочные эффекты отмечались у $25 \%$ пациентов. И только у 5\% пациентов, получавших Мильгамму, отмечались побочные эффекты. Авторы заключили, что комбинированная терапия Мильгаммой и диклофенаком дает более выраженный и быстрый обезболивающий эффект, чем монотерапия. При этом монотерапия Мильгаммой может также применяться при острой боли в спине в качестве обезболивающей терапии. Преимущества монотерапии Мильгаммой перед монотерапией диклофенаком - низкая частота развития побочных эффектов. Авторы предположили применение комбинированной терапии у пациентов с интенсивной (8-10 баллов по ВАШ) острой ПНБ, когда необходимо быстро получить обезболивающий эффект. Также авторы сформулировали гипотезу о том, что при комбинированном назначении НПВС и препарата Мильгамма возможно применение меньших доз НПВС. Однако подтверждение данной гипотезы требует проведения соответствующих клинических исследований. 


\section{ЗАКЛЮЧЕНИЕ}

Эффективность комбинации НПВС и витаминов группы В в лечении острой неспецифической ПКБ продолжает изучаться. В настоящее время В нашей стране проводится крупное многоцентровое (25 лечебных учреждений) исследование эффективности и безопасности дополнительной терапии препаратами Мильгамма и Мильгамма композитум в рутинной практике у пациентов с острой неспецифической ПКБ, получающих современные НПВС. Информация об исследовании представлена в международном реестре www.clinicaltrials.gov Национального института здоровья США. Результаты данного исследования планируется опубликовать в 2020 г.

Таким образом, острая неспецифическая ПКБ - распространенное заболевание с благоприятным прогнозом. К основным принципам эффективного лечения острой неспецифической ПКБ относятся информирование пациента о благоприятном прогнозе заболевания, поддержа- ние повседневной активности и оптимальная фармакотерапия для облегчения боли. При острой неспецифической ПКБ эффективны препараты НПВС в комбинации или без миорелаксантов. Новое направление в лечении острой неспецифической ПКБ - применение НПВС в комбинации с препаратами витаминов группы В. В данной комбинированной фармакотерапии витамины группы В рассматриваются как коанальгетики. По результатам клинических исследований комбинированная терапия НПВС и витаминами группы В позволяет сократить продолжительность приема НПВС и снизить риски развития побочных эффектов НПВС. Среди препаратов, содержащих витамины группы B, наиболее широко в России применяется Мильгамма, эффективность и безопасность которой при острой неспецифической ПКБ продемонстрирована в нескольких российских клинических исследованиях.

Поступила / Received 14.10.2019 Отрецензирована / Review 31.10.2019 Принята в печать / Accepted 04.11.2019

\section{Список литературы}

1. Qaseem A., Wilt T.J., McLean R.M., Forciea M.A. Clinical Guidelines Committee of the American College of Physicians. Noninvasive Treatments for Acute, Subacute, and Chronic Low Back Pain: A Clinical Practice Guideline From the American College of Physicians. Ann Intern Med. 2017;166(7):514-530. doi: 10.7326/ M16-2367.

2. Парфенов В.А., Головачева В.А. Диагностика и лечение острой неспецифической пояснично-крестцовой боли. Терапевтический архив. 2019;91(8):155-159. doi: 10.26442/004 03660.2019.08.000315.

3. Friedly J., Standaert C., Chan L. Epidemiology of spine care: the back pain dilemma. Physical medicine and rehabilitation clinics of North America. Phys Med Rehabil Clin N Am. 2010;21(4):659-77. doi: 10.1016/j. pmr.2010.08.002

4. Tavee J.O., Levin K.H. Low Back Pain. Continuum. 2017;23(2):467-486. doi: 10.1212/ CON.0000000000000449.

5. Bardin L.D., King P., Maher C.G. Diagnostic triage for low back pain: a practical approach for primary care. Med J Aust. 2017;206(6):268273. doi: 10.5694/mja16.00828.

6. Chou R., Qaseem A., Snow V., Casey D., Cross J.T. Jr., Shekelle P., Owens D.K. Clinical Efficacy Assessment Subcommittee of the American College of Physicians; American College of Physicians; American Pain Society Low Back Pain Guidelines Panel. Diagnosis and treatment of low back pain: a joint clinical practice guideline from the American College of Physicians and the American Pain Society. Ann Intern Med. 2007;(147):478-479. doi: 10.7326/0003-4819-147-7-200710020-00006.

7. Головачева В.А., Голубева В.В. Как помочь пациенту с хронической неспецифической болью в нижней части спины? РМЖ. 2014;(16):1163-1167. https://www.rmj.ru/articles/nevrologiya/Kak_pomochy_pacientu_s_ hronicheskoy_nespecificheskoy_bolyyu_v_ nighney_chasti_spiny/.

8. Парфенов В.А., Яхно Н.Н., Кукушкин М.Л., Чурюканов М.В., Давыдов О.С., Головачева В.А., Исайкин А.И., Ачкасов Е.Е., Евзиков Г.Ю., Каратеев А.Е., Хабиров Ф.А., Широков В.А. Острая неспецифическая (скелетномышечная) поясничная боль. Рекомендации Российского общества по изучению боли
(РОИБ). Неврология, нейропсихиатрия, психосоматика. 2018;10(2):4-11.

9. Подчуфарова Е.В., Яхно Н.Н. Боль в спине. М., 2010. 368 c.

10. Парфенов В.А., Исайкин А.И. Боли в нижней части спины: мифы и реальность. М.: ИМАПРЕСС; 2016, 104 с.

11. Парфенов В.А., Исайкин А.И. Боли в поясничной области. Библиотека практического врача. М.: МЕДпрес-информ; 2018, 200 с.

12. Парфенов В.А., Головачева В.А. Хроническая боль и ее лечение в неврологии. М.: ГЭОТАРМедиа; 2018. 288 с.

13. Low back pain and sciatica in over $16 \mathrm{~s}$ : assessment and management. NICE guideline [NG59]. Published date: November 2016. Available at: www.nice.org.uk/guidance/ng59/ evidence.

14. Stochkendahl M.J., Kjaer P., Hartvigsen J., Kongsted A., Aaboe J., Andersen M., et al. National Clinical Guidelines for non-surgical treatment of patients with recent onset low back pain or lumbar radiculopathy. Eur Spine J. 2018;27(1):60-75. doi: 10.1007/s00586-0175099-2

15. Oliveira C.B., Maher C.G., Pinto R.Z., Traeger A.C., Lin C.C., Chenot J.F., van Tulder M., Koes B.W. Clinical practice guidelines for the management of non-specific low back pain in primary care: an updated overview. Eur Spine J. 2018;27(11):2791-2803. doi: 10.1007/s00586018-5673-2.

16. Koes B.W., van Tulder M., Lin C.W., Macedo L.G., McAuley J., Maher C. An updated overview of clinical guidelines for the management of non-specific low back pain in primary care. Eur Spine J. 2010;19(12):2075-2094. doi: 10.1007/s00586-010-1502-y.

17. van Tulder M.W., Touray T., Furlan A.D., Solway S., Bouter L.M. Muscle relaxants for non-specific low back pain. Cochrane Database Syst Rev. 2003;(2):CD004252. doi: 10.1002/14651858.CD004252.

18. Pareek A., Chandurkar N., Chandanwale A.S., Ambade R., Gupta A., Bartakke G. Aceclofenactizanidine in the treatment of acute low back pain: a double-blind, double-dummy, randomized, multicentric, comparative study against aceclofenac alone. Eur Spine J. 2009;18(12):1836-1842. doi: 10.1007/s00586009-1019-4.
19. Friedman B.W., Dym A.A., Davitt M., Holden L., Solorzano C., Esses D., Bijur P.E., Gallagher E.J. Naproxen with cyclobenzaprine, oxycodone/ acetaminophen, or placebo for treating acute low back pain: a randomized clinical trial. JAMA. 2015;314(15):1572-1580. doi: 10.1001/ jama.2015.13043.

20. Wong JJ., Côté P., Sutton D.A., Randhawa K., Yu H., Varatharajan S., et al. Clinical practice guidelines for the noninvasive management of low back pain: A systematic review by the Ontario Protocol for Traffic Injury Management (OPTIMa) Collaboration. Eur J Pain. 2017;21(2):201-216. doi: 10.1002/ejp.931.

21. Хабиров Ф.А. Руководство по клинической неврологии позвоночника. Казань: Медицина; 2006. 520 c.

22. Хабиров Ф.А., Хабирова Ю.Ф. Боль в шее и спине: руководство для врачей. Казань: Медицина; 2014. 504 с.

23. Камчатнов П.Р. Повышение эффективности и безопасности лечения пациентов с поясничной болью. Журнал неврологии и психиатрии им. С.С. Корсакова. 2016;116(10):28-33. doi: 10.17116/jnevro201611610128-33.

24. Журавлева М.В., Ших Е.В., Махова Л.А. Связь анальгетического и нейротропного эффектов на примере мильгаммы. Терапевтический архив. 2012;84(12):131-134. Режим доступа: http://www.medlib.am/ content/ter_arxiv_2012_12.pdf.

25. Woelk H., Lehrl S., Bitsch R., Kopcke W. Benfotiamine in treatment of alcoholic polyneuropathy: an 8-week randomized controlled study (BAP I Study). Alcohol Alcohol. 1998;33(6):631638. doi: 10.1093/alcalc/33.6.631.

26. Емельянова А.Ю., Зиновьева О.Е. Патогенез и лечение полиневропатий: роль витаминов группы В. Эффективная фармакотерапия. Неврология. 2015;(40):42-48. Режим доступа: http://umedp.ru/upload/iblock/e3e/ nevro_05_2015.pdf.

27. Briani C., Dalla Torre C., Citton V., Manara R., Pompanin S., Binotto G., Adami F. Cobalamin deficiency: clinical picture and radiological findings. Nutrients. 2013;5(11):4521-45239. doi: 10.3390/nu5114521.

28. Парфенов В.А., Головачева В.А., Фадеев В.В., Воловик А.Ю., Головачева А.А. Оптимизация ведения пациентов с диабетической полиневропатией с помощью междисциплинар- 
ного подхода. Медицинский Совет. 2017;(1S):71-79. doi: 10.21518/2079-701X2017-0-71-79.

29. Ang C.D., Alviar M.J., Dans A.L., Bautista-Velez G.G., Villaruz-Sulit M.V., Tan J.J., Co H.U., Bautista M.R., Roxas A.A. Vitamin B for treating peripheral neuropathy. Cochrane Database Syst Rev. 2008;(3):CD004573. doi: 10.1002/14651858.CD004573.pub3.

30. Jayabalan B., Low L.L. Vitamin B supplementation for diabetic peripheral neuropathy. Singapore Med J. 2016;57(2):55-59. doi: 10.11622/smedj.2016027.

31. Nardone R., Höller Y., Storti M., Christova M., Tezzon F., Golaszewski S., Trinka E., Brigo F. Thiamine Deficiency Induced Neurochemical, Neuroanatomical, and Neuropsychological Alterations: A Reappraisal. Scientific World Journal. 2013:309143. doi: 10.1155/2013/309143.

32. Scott J., Molloy A. The discovery of vitamin B (12). Ann Nutr Metab. 2012;61(3):239-245. doi: 10.1159/000343114.

33. Mooney S., Leuendorf J.E., Hendrickson C., Hellmann H. Vitamin B6: a long known compound of surprising complexity. Molecules. 2009:14(1):329-351. doi: 10.3390/molecules14010329.

34. Kobzar G., Mardla V., Rätsep I., Samel N. Effect of vitamin $B(6)$ vitamers on platelet aggregation. Platelets. 2009;20(2):120-124. doi: 10.1080/09537100802687674.

35. Amir J., Ginat S., Cohen Y.H., Marcus T.E., Keller N., Varsano I. Lidocaine as a diluent for administration of benzathine penicillin $\mathrm{G}$. Pediatr Infect Dis J. 1998;17(10):890-893. doi: 10.1097/00006454-199810000-00008.

36. França D.S., Souza A.L., Almeida K.R., Dolabella S.S., Martinelli C., Coelho M.M. B vitamins induce an antinoceceptive effect in the acetic acid and formaldehyde models of nociception in mice. Eur J Pharmacol. 2001;421(3):157-164. oi: 10.1016/s0014-2999(01)01038-x.

37. Sánchez-Ramírez G.M., Caram-Salas N.L., Rocha-González H.I., Vidal-Cantú G.C., MedinaSantillán R., Reyes-García G., Granados-Soto V. Benfotiamine relieves inflamatory and neuropathic pain in rats. Eur J Pharmacol. 2006;530(1-2):48-53. doi: 10.1016/j. ejphar.2005.11.016.

38. Moallem S.A., Hosseeinzaden H., Farahi S. A study of acute and chronic anti-nociceptive and anti-inflammatory effects of thiamine in mice. Iran Biomed J. 2008:12(3):173-178. Available at: http://ibj.pasteur.ac.ir/browse php?a_id $=84 \&$ sid=1\&slc_lang $=e n$.

39. Bartoszyk G.D. The interactions of vitamins B1, B6 and B12 with non-steroidal antirheumatic and analgesic drugs: animal experiment results. Klin Wochenschr. 1990;68(2):121-4. doi: 10.1007/bf01646859.

40. Bartoszyk G.D., Wild A. B-vitamins potentiate the antinociceptive effect of diclofenac in carrageenin-induced hyperalgesia in the rat tail pressure test. Neurosci Lett. 1989;101(1):95100. doi: 10.1016/0304-3940(89)90447-3.

41. Jurna I., Carlsson K.H., Komen W., Bonke D. Acute effects of vitamin B6 and fixed combinations of vitamin B1, B6 and B12 on nociceptive activity evoked in the rat thalamus: dose - response relationship and combinations with morphine and paracetamol. Klin Wochenschr. 1990;68(2):129-135. doi: 10.1007/ bf01646861.

42. Kuhlwein A., Meyer H.J., Koehler C.O. Reduced need for diclofenac with concomitant B-vitamin therapy: Results of a double-blind clinical study with re-duced diclofenac-dosage $(75 \mathrm{mg}$ diclofenac vs. $75 \mathrm{mg}$ diclofenac plus B-vitamins daily) in pa- tients with acute lumbago. Klin Wochenschr. 1990;68(2):107-115 doi: $10.1007 / b f 01646857$.

43. Vetter G., Brüggemann G., Lettko M., Schwieger G., Asbach H., Biermann W., Bläsius K., et al. Shortening diclofenac therapy by B vitamins. Results of a randomized double-blind study, diclofenac $50 \mathrm{mg}$ versus diclofenac $50 \mathrm{mg}$ plus $B$ vitamins, in painful spinal diseases with degenerative changes. $Z$ Rheumatol. 1988;47(5):351-362. Available at: https://www. ncbi.nlm.nih.gov/pubmed/?term=Vetter+G\%2C + Br\%C3\%BCggemann+G\%2C+Lettko+M.

44. Bruggemann G., Koehler C.O., Koch E.M. Results of a double-blind study of diclofenac + vitamin B1, B6, B12 versus diclofenac in patients with acute pain of the lumbar vertebrae. A multicenter study. Klin Wochenschr. 1990;68(2):116-120. doi: 10.1007/bf01646858

45. Lettko M. Ergebnisse einer Doppelblindstudie, Diclofenac und B-vitamine gegen Diclofenac, zur Priifung der additiven Wirksamkeit der B-Vitamine. Rheuma, Schmerz \& Entzundung. 1986;(8):22-30. Available at: https://ink. springer.com/chapter/10.1007/978-3-642-72427-5 14.

46. Mibielli M.A., Geller M., Cohen J.C.', Goldberg S.G., Cohen M.T., Nunes C.P., Oliveira L.B., da Fonseca A.S. Diclofenac Plus B Vitamins Versus Diclofenac Monotherapy in Lumbago: the DOLOR Study. Curr Med Res Opin. 2009;25(11):2589-2599. doi: 10.3111/13696990903246911

47. Данилов А.Б. Лечение острой боли в спине витамины группы «В» или НПВП? РМЖ. 2010;(0):35-40. Режим доступа: https://www. rmj.ru/articles/bolevoy_sindrom/Lechenie ostroy_boli_v_spine_vitaminy_gruppy_V_i $\bar{i}_{\text {_ }}$ NPVP/.

\section{References}

1. Qaseem A., Wilt T.., McLean R.M., Forciea M.A. Clinical Guidelines Committee of the American College of Physicians. Noninvasive Treatments for Acute, Subacute, and Chronic Low Back Pain: A Clinical Practice Guideline From the American College of Physicians. Ann Intern Med. 2017;166(7):514-530. doi: 10.7326/M16-2367.

2. Parfenov V.A., Golovacheva V.A. Diagnosis and treatment of acute low back pain. Therapeutic Archive. 2019;91(8):155-159. (In Russ.) doi: 10. 26442/00403660.2019.08.000315.

3. Friedly J., Standaert C., Chan L. Epidemiology of spine care: the back pain dilemma. Physical medicine and rehabilitation clinics of North America. Phys Med Rehabil Clin N Am. 2010;21(4):659-77. doi: 10.1016/j. pmr.2010.08.002

4. Tavee J.O., Levin K.H. Low Back Pain. Continuum. 2017;23(2):467-486. doi: 10.1212/ CON.0000000000000449.

5. Bardin L.D., King P., Maher C.G. Diagnostic triage for low back pain: a practical approach for primary care. Med J Aust. 2017;206(6):268273. doi: $10.5694 / \mathrm{mja} 16.00828$

6. Chou R., Qaseem A., Snow V., Casey D., Cross J.T. Jr., Shekelle P., Owens D.K. Clinical Efficacy Assessment Subcommittee of the American College of Physicians; American College of Physicians; American Pain Society Low Back Pain Guidelines Panel. Diagnosis and treatment of low back pain: a joint clinical practice guideline from the American College of Physicians and the American Pain Society. Ann Intern Med. 2007;(147):478-479. doi: 10.7326/0003-4819-147-7-200710020-00006.

7. Golovacheva V.A., Golubeva V.V. How to help patients with chronic nonspecific low back pain? RMZH = RMJ. 2014;(16):1163-1167 (In Russ.) Available at: https://www.rmj.ru/articles/nevrologiya/Kak_pomochy_pacientu_s_ hronicheskoy_nespecificheskoy_bolyyu_v_ nighney_chasti_spiny/.

8. Parfenov V.A., Yakhno N.N., Kukushkin M.L., et al. Acute nonspecific (musculoskeletal) low back pain. Guidelines of the Russian Society for the Study of Pain (RSSP). Nevrologiya, neiropsikhiatriya, psikhosomatika $=$ Neurology, neuropsychiatry, psychosomatics. 2018;10(2):4-11. (In Russ.) doi: 10.14412/2074-2711-2018-2-4-11.

9. Podchufarova EV, Yakhno NN. Pain in back. Moscow; 2010.368 p. (In Russ.)

10. Parfenov V.A., Isaykin A.I. Low back pain: legends and reality. Moscow: IMA-PRESS; 2016, 104 p. (In Russ.)

11. Parfenov V.A., Isaykin A.I. Pain in the lumbar region. General Practitioner's Library. Moscow: MEDpres-inform; 2018, 200 p. (In Russ.)

12. Parfenov V.A., Golovacheva V.A. Chronic pain and its treatment in neurology. Moscow: GEOTAR-Media; 2018. 288 p. (In Russ.)

13. Low back pain and sciatica in over 16s: assessment and management. NICE guideline [NG59]. Published date: November 2016. Available at: www.nice.org.uk/guidance/ng59/evidence.

14. Stochkendahl M.J., Kjaer P., Hartvigsen J., Kongsted A., Aaboe J., Andersen M., et al. National Clinical Guidelines for non-surgical treatment of patients with recent onset low back pain or lumbar radiculopathy. Eur Spine J. 2018;27(1):60-75. doi: 10.1007/s00586-0175099-2

15. Oliveira C.B., Maher C.G., Pinto R.Z., Traeger A.C., Lin C.C., Chenot J.F., van Tulder M., Koes B.W. Clinical practice guidelines for the man- agement of non-specific low back pain in primary care: an updated overview. Eur Spine J. 2018;27(11):2791-2803. doi: 10.1007/s00586018-5673-2.

16. Koes B.W., van Tulder M., Lin C.W., Macedo L.G., McAuley J., Maher C. An updated overview of clinical guidelines for the management of non-specific low back pain in primary care. Eur Spine J. 2010;19(12):2075-2094. doi: 10.1007/s00586-010-1502-y.

17. van Tulder M.W., Touray T., Furlan A.D., Solway S., Bouter L.M. Muscle relaxants for non-specific low back pain. Cochrane Database Syst Rev. 2003;(2):CD004252. doi: 10.1002/14651858. CD004252.

18. Pareek A., Chandurkar N., Chandanwale A.S., Ambade R., Gupta A., Bartakke G. Aceclofenactizanidine in the treatment of acute low back pain: a double-blind, double-dummy, randomized, multicentric, comparative study against aceclofenac alone. Eur Spine J. 2009;18(12):1836-1842. doi: 10.1007/s00586009-1019-4.

19. Friedman B.W., Dym A.A., Davitt M., Holden L., Solorzano C., Esses D., Bijur P.E., Gallagher E.J. Naproxen with cyclobenzaprine, oxycodone/ acetaminophen, or placebo for treating acute low back pain: a randomized clinical trial. JAMA. 2015;314(15):1572-1580. doi: 10.1001/ jama.2015.13043.

20. Wong J.J., Côté P., Sutton D.A., Randhawa K., Yu H., Varatharajan S., et al. Clinical practice guidelines for the noninvasive management of low back pain: A systematic review by the Ontario Protocol for Traffic Injury Management (OPTIMa) Collaboration. Eur J Pain. 2017;21(2):201-216. doi: 10.1002/ejp.931. 
21. Khabirov FA. Guidelines for clinical neurology of the spine. Kazan: Medicine; 2006. 520 p. (In Russ.)

22. Khabirov F.A., Khabirova Yu.F. Back and neck pain: a guide for practitioners. Kazan: Medicine 2014.504 p. (In Russ.)

23. Kamchatnov P.R. Improvement of the efficacy and safety of the treatment of patients with low back pain. Zhurnal nevrologii i psikhiatrii imeni S.S. Korsakova = S.S. Korsakov Journal of Neurology and Psychiatry. 2016;116(10):28-33. (In Russ.). doi: 10.17116/jnevro201611610128-33.

24. Zhuravleva M.V., Shikh E.V., Makhova A.A. A relationship between analgesic and neuro tropic effects by the example of milgamma. Terapevticheskiy arkhiv = Therapeutic archive 2012;84(12):131-134. (In Russ.) Available at: http://www.medlib.am/content/ter_ arxiv 2012 12.pdf.

25. Woelk H., Lêhrl S., Bitsch R., Kopcke W. Benfotiamine in treatment of alcoholic polyneuropathy: an 8-week randomized controlled study (BAP I Study). Alcohol Alcohol. 1998;33(6):631638. doi: 10.1093/alcalc/33.6.631.

26. Yemelyanova A.Yu., Zinovyeva O.Ye. Pathogenesis and Treatment of Polyneuropathies: a Role for B Vitamins. Ehffektivnaya farmakoterapiya. Nevrologiya $=$ Effective Pharmacotherapy. 2015;(40):42-48. (In Russ.) Available at: http://umedp.ru/upload/ iblock/e3e/nevro_05_2015.pdf.

27. Briani C., Dalla Torre C., Citton V., Manara R., Pompanin S., Binotto G., Adami F. Cobalamin deficiency: clinical picture and radiological findings. Nutrients. 2013;5(11):4521-45239. doi: $10.3390 /$ nu5114521.

28. Parfenov V.A., Golovacheva V.A., Fadeev V.V., Volovik A.Y., Golovacheva A.A. Optimization diabetic polyneuropathy patients management using a multidisciplinary approach. Meditsinskiy sovet $=$ Medical Council. 2017;(1S):71-79. (In Russ.) doi: 10.21518/2079-701X-2017-0-71-79.

29. Ang C.D., Alviar M.J., Dans A.L., Bautista-Velez G.G., Villaruz-Sulit M.V., Tan J.J., Co H.U., Bautista M.R., Roxas A.A. Vitamin B for treating peripheral neuropathy. Cochrane Database Syst Rev. 2008;(3):CD004573. doi: 10.1002/14651858.CD004573.pub3.

30. Jayabalan B., Low L.L. Vitamin B supplementation for diabetic peripheral neuropathy.
Singapore Med J. 2016;57(2):55-59. doi 10.11622/smedj.2016027.

31. Nardone R., Höller Y., Storti M., Christova M., Tezzon F., Golaszewski S., Trinka E., Brigo F. Thiamine Deficiency Induced Neurochemical, Neuroanatomical, and Neuropsychological Alterations: A Reappraisal. Scientific World Journal. 2013:309143. doi: 10.1155/2013/309143.

32. Scott J., Molloy A. The discovery of vitamin B (12). Ann Nutr Metab. 2012;61(3):239-245. doi: 10.1159/000343114.

33. Mooney S., Leuendorf J.E., Hendrickson C., Hellmann H. Vitamin B6: a long known compound of surprising complexity. Molecules. 2009;14(1):329-351. doi: 10.3390/molecules14010329.

34. Kobzar G., Mardla V., Rätsep I., Samel N. Effect of vitamin $B(6)$ vitamers on platelet aggregation. Platelets. 2009;20(2):120-124. doi: 10.1080/09537100802687674.

35. Amir J., Ginat S., Cohen Y.H., Marcus T.E., Keller N., Varsano I. Lidocaine as a diluent for administration of benzathine penicillin $\mathrm{G}$. Pediatr Infect Dis J. 1998;17(10):890-893. doi: 10.1097/00006454-199810000-00008.

36. França D.S., Souza A.L., Almeida K.R., Dolabella S.S., Martinelli C., Coelho M.M. B vitamins induce an antinoceceptive effect in the acetic acid and formaldehyde models of nociception in mice. Eur J Pharmacol. 2001;421(3):157-164. doi: 10.1016/s0014-2999(01)01038-x.

37. Sánchez-Ramírez G.M., Caram-Salas N.L., Rocha-González H.I., Vidal-Cantú G.C., MedinaSantillán R., Reyes-García G., Granados-Soto V. Benfotiamine relieves inflamatory and neuropathic pain in rats. Eur J Pharmacol. 2006;530(12):48-53. doi: 10.1016/j.ejphar.2005.11.016.

38. Moallem S.A., Hosseeinzaden H., Farahi S. A study of acute and chronic anti-nociceptive and anti-inflammatory effects of thiamine in mice. Iran Biomed J. 2008;12(3):173-178. Available at: http://ibj.pasteur.ac.ir/browse php?a_id=84\&sid=1\&slc_lang=en.

39. Bartoszyk G.D. The interactions of vitamins B1, B6 and B12 with non-steroidal antirheumatic and analgesic drugs: animal experiment results. Klin Wochenschr. 1990;68(2):121-4. doi: 10.1007/bf01646859.

40. Bartoszyk G.D., Wild A. B-vitamins potentiate the antinociceptive effect of diclofenac in carrageenin-induced hyperalgesia in the rat tail pressure test. Neurosci Lett. 1989;101(1):95100. doi: 10.1016/0304-3940(89)90447-3.

41. Jurna I., Carlsson K.H., Komen W., Bonke D. Acute effects of vitamin B6 and fixed combinations of vitamin B1, B6 and B12 on nociceptive activity evoked in the rat thalamus: dose response relationship and combinations with morphine and paracetamol. Klin Wochenschr. 1990;68(2):129-135. doi: 10.1007/bf01646861.

42. Kuhlwein A., Meyer H.J., Koehler C.O. Reduced need for diclofenac with concomitant B-vitamin therapy: Results of a double-blind clinical study with re-duced diclofenac-dosage $(75 \mathrm{mg}$ diclofenac vs. $75 \mathrm{mg}$ diclofenac plus B-vitamins daily) in pa-tients with acute lumbago. Klin Wochenschr. 1990;68(2):107-115. doi: $10.1007 /$ bf01646857.

43. Vetter G., Brüggemann G., Lettko M., Schwieger G., Asbach H., Biermann W., Bläsius K., et al. Shortening diclofenac therapy by B vitamins. Results of a randomized double-blind study, diclofenac $50 \mathrm{mg}$ versus diclofenac $50 \mathrm{mg}$ plus $B$ vitamins, in painful spinal diseases with degenerative changes. $Z$ Rheumatol. 1988;47(5):351-362. Available at: https://www. ncbi.nlm.nih.gov/pubmed/?term=Vetter+G\%2C $+\mathrm{Br} \% \mathrm{C} 3 \%$ BCggemann+G\%2C+Lettko+M.

44. Bruggemann G., Koehler C.O., Koch E.M. Results of a double-blind study of diclofenac + vitamin B1, B6, B12 versus diclofenac in patients with acute pain of the lumbar vertebrae. A multicenter study. Klin Wochenschr. 1990;68(2):116-120. doi: 10.1007/bf01646858.

45. Lettko M. Ergebnisse einer Doppelblindstudie, Diclofenac und B-vitamine gegen Diclofenac, zur Priifung der additiven Wirksamkeit der B-Vitamine. Rheuma, Schmerz \& Entzundung. 1986;(8):22-30. Available at: https://ink.springer. com/chapter/10.1007/978-3-642-72427-5 14.

46. Mibielli M.A., Geller M., Cohen J.C., Goldberg S.G., Cohen M.T., Nunes C.P., Oliveira L.B., da Fonseca A.S. Diclofenac Plus B Vitamins Versus Diclofenac Monotherapy in Lumbago: the DOLOR Study. Curr Med Res Opin. 2009;25(11):2589-2599. doi: $10.3111 / 13696990903246911$.

47. Danilov A.B. Treatment of acute back pain: B-vitamins or NSAIDs? RMZH $=$ RMJ. 2010;(0):35-40. (In Russ.) Available at: https:// www.rmj.ru/articles/bolevoy_sindrom/ Lechenie_ostroy_boli_v_spine_vitaminy gruppy_V_ili_NPVP/.

\section{Информация об авторах:}

Головачева Вероника Александровна, к.м.н., ассистент кафедры нервных болезней и нейрохирургии лечебного факультета Института клинической медицины, Федеральное государственное автономное образовательное учреждение высшего образования «Первый Московский государственный медицинский университет имени И.М. Сеченова» Министерства здравоохранения Российской Федерации (Сеченовский Университет); 119991, Россия, Москва, ул. Трубецкая, д. 8, стр. 2; e-mail: xoxo.veronicka@gmail.com

Парфенов Владимир Анатольевич, д.м.н., профессор, заведующий кафедрой нервных болезней и нейрохирургии лечебного факультета Института клинической медицины, Федеральное государственное автономное образовательное учреждение высшего образования «Первый Московский государственный медицинский университет имени И.М. Сеченова» Министерства здравоохранения Российской Федерации (Сеченовский Университет); 119991, Россия, Москва, ул. Трубецкая, д. 8, стр. 2

\section{Information about the authors:}

Veronika A. Golovacheva, Cand. of Sci. (Med.), Teaching Assistant, Chair for Nervous Diseases and Neurosurgery, Faculty of General Medicine, Federal State Autonomous Educational Institution of Higher Education «Sechenov First Moscow State Medical University» of the Ministry of Health of the Russian Federation (Sechenov University); 8, Bldg. 2, Trubetskaya St., Moscow, 119991, Russia ; e-mail: xoxo.veronicka@gmail.com Vladimir A. Parfenov, Dr. of Sci. (Med.), Professor, Head of Chair for Nervous Diseases and Neurosurgery, Faculty of General Medicine, Federal State Autonomous Educational Institution of Higher Education «Sechenov First Moscow State Medical University» of the Ministry of Health of the Russian Federation (Sechenov University); 8, Bldg. 2, Trubetskaya St., Moscow, 119991, Russia 8, Bldg. 2, Trubetskaya St., Moscow, 119991, Russia 